clinic for investigation of secondary infertility and had given a six month history of oligomenorrhoea and galactorrhoea. Her serum prolactin concentration had then been $1820 \mathrm{mU} / 1$ (normal $\leqslant 360 \mathrm{mU} / \mathrm{l}$ ), and radiographs of the pituitary fossa had shown nothing abnormal. Thyroid function tests had not been performed. The patient had been treated with conventional doses of bromocriptine for four months and the galactorrhoea had stopped, the prolactin concentration falling to $140 \mathrm{mU} /$. Her periods had returned to normal and she had become pregnant and given birth to a normal child in 1982. After a nursing period of about five months intermittent secretion of milk had persisted until the patient was seen in our clinic in 1985.

She denied significant menstrual irregularities and she was taking no medication. On examination bilateral galactorrhoea was confirmed and, apart from a slowish pulse of $66 / \mathrm{min}$, she was clinically euthyroid. Serum prolactin concentration was again high at 1360 $\mathrm{mU} / \mathrm{l}$. Her serum thyroxine value was low at $16 \mathrm{nmol} / \mathrm{l}$ $(1.25 \mu \mathrm{g} / 100 \mathrm{ml})$ with serum thyroid stimulating hormone values greater than $45 \mathrm{mU} / 1$, a picture compatible with primary hypothyroidism. Thyroid microsomal autoantibodies were present at a titre of $1 / 3200$. Computed tomography showed a large pituitary gland, probably hyperplastic, without focal abnormalities. She was treated with thyroxine $0.05 \mathrm{mg}$ daily, increasing to $0.1 \mathrm{mg}$ daily, and within three months her serum thyroxine, thyroid stimulating hormone, and prolactin concentrations returned to normal and the galactorrhoea stopped.

We concluded that this patient's hyperprolactinaemia was associated with her primary hypothyroidism and that the stimulating effect of the thyrotrophin releasing hormone on the lactotrophs had been counteracted by the prolactin inhibiting action of the dopamine agonist (bromocriptine) administered for what was thought to be idiopathic hyperprolactinaemia. The restoration of fertility after the return to normal of serum prolactin concentrations suggests that hyperprolactinaemia might play a part in the infertility commonly associated with primary hypothyroidism. Raised serum prolactin values are common in primary hypothyroidism ( $39 \%$ in a series of 49 patients), but galactorrhoea is much less common and appears to occur mostly in women with a history of pregnancy. ${ }^{12}$ In 235 patients investigated for galactorrhoea 10 had primary hypothyroidism but only five had raised prolactin values. ${ }^{3}$ It is therefore important to assess thyroid state not only in patients with hyperprolactinaemia but in all patients with galactorrhoea, including, of course, those who are clinically euthyroid.

I am grateful to Dr J Q Matthias, for permission to report on a patient under his care.

C Christopoulos

St Ann's Hospital

London N15

1 Honbo KS, Van Herle AJ, Kellett KA. Serum prolactin levels in untreated primary hypothyroidism. Am J Med 1978;64:782-7. 2 Onishi T, Miyai K, Aono T, et al. Primary hypothyroidism and galactorrhoea. Am J Med 1977;63:373-8.

3 Kleinberg DL, Noel GL, Frantz AG. Galactorrhoea: a study of 235 cases, including 48 with pituitary tumors. $N$ Engl 3 Med 1977;296:589-600.

\section{Neurological complications of coronary artery bypass surgery}

SIR,-The six month follow up paper from Newcastle on the neurological complications of coronary artery bypass graft surgery (19 July, p 165 ) is reassuring only in that the high incidence of cerebral changes, seen early after surgery, proved to be largely recoverable by six months. Dr Pamela $\mathrm{J}$ Shaw and her colleagues are to be congratulated on their assiduous follow up. A comparable improvement has been found in our smaller group of patients followed up for two months after surgery. ${ }^{1}$ While the six month results are comforting at first sight, detection of impaired function soon after operation has now been shown to carry serious long term implications despite apparent complete recovery. ${ }^{2}$

Because of the descriptive nature of the study i is difficult to use their data to improve practice. I is tempting to relate the "soft" neurological signs and neuropsychological impairment, seen in so many patients, to inadequate perfusion while on bypass but before this apparently obvious inference can be drawn information is needed about patients undergoing other types of major surgery. Our own preliminary data, including a contro group, ${ }^{1}$ show that neuropsychological changes are seen after thoracotomy and aortoiliac surgery. I would be valuable to know what Dr Shaw and her colleagues found in control cases so that the precise role of cardiopulmonary bypass and cardiac surgery can be assessed.

A relatively large number of the Newcastle patients developed peripheral nerve lesions. Al though a specific mononeuritis related to bypass has been proposed, ${ }^{3}$ it seems more likely that popliteal and ulnar nerve lesions, for example, relate to mechanical factors. ${ }^{4}$ Brachial plexus lesions, which they also include, are a recognised complication of cardiac surgery ${ }^{56}$ which may resolve and which are also encountered after thymectomy, confirming that it is the sternotomy not the bypass, that is to blame.

The most serious complication proved to be stroke, which is generally believed to be embolic though hypoperfusion may, of course, increase the brain's vulnerability to embolism. It would be helpful to know whether the clinical picture and computed tomography suggested that perioperative stroke was due to infarction in the watershed areas or in the territory of the branches of the middle cerebral artery.

TOM TREASURE

MICHAEL HARRISON

STANTON NEWMAN

Peter SMIth

Middlesex Hospital,
London W1N 8AA

1 Smith PLC, Treasure T, Newman SP, et al. Cerebral consequences of cardiopulmonary bypass. Lancet 1986;i:823

2 Sotaniemi KA, Mononen H, Hokkanen TE. Long-term cerebral outcome after open-heart surgery. A five-year neuropsychological follow-up study. Stroke 1986;17:410-6.

3 Keates JRW, Innocenti DM, Ross DN. Mononeuritis multiplex: a complication of open heart surgery. F Thorac Cardiovasc Surg 1975:69:816-9.

4 Winner JB Harrison MJG. Iatrogenic nerve injury Pustgrad Med J 1982;58:142-5

5 Treasure T, Garnett $R, O^{\prime}$ Connor J, Treasure JL. Injury of the lower trunk of the brachial plexus as a complication of median sternotomy for cardiac surgery. Ann R Coll Surg Engl 1980;62: 378 .

6 Treasure T. Brachial plexus injury due to median sternotomy. Thorax 1981;36:80.

AUTHORS' REPLY - We agree that the five yea neuropsychological outcome in patients undergoing heart valve surgery, reported by Sotaniemi et $a l,{ }^{1}$ is a cause for concern. The course of postoperative complications may differ in patients undergoing coronary bypass, and we intend to follow up our cohort to assess long term outcome. The assessment of the functional impact of any detectable long term disorders will also be of great interest.

We agree that before neurological and neuro psychological complications can be attributed to cardiopulmonary bypass per se it is important to evaluate the effects of major surgery without the use of extracorporeal circulation. In the Newcastle study we compared the findings in the 312 patients who underwent coronary bypass with those in a group of 50 patients undergoing major non-cardiac vascular surgery. We found a substantially lower incidence of complications in control patients than that reported by Smith et $a l,{ }^{2}$ and details of the study will shortly be submitted for publication.

Computed tomograms were obtained in four of the 15 patients who developed stroke in our study. In three of the four the scan appearances suggested embolic rather than watershed infarction. In all the patients with severely disabling major stroke the brunt of the damage was borne by the right hemisphere. It is possible that the right carotid artery may be more liable to embolisation, being the first major branch of the ascending aorta. Others have found that the right hemisphere is more likely to be injured during heart surgery. ${ }^{3}$

Our analysis of causative and predisposing factors for neurological and neuropsychological complications, including stroke, is currently in progress. As others have found, it is usually difficult to pinpoint the cause for stroke in individual patients. ${ }^{4}$

Though embolic events have been considered to be the major cause of stroke during heart surgery, hypoperfusion may also be a contributory factor.

Further work is needed to define patterns of cerebral blood flow during heart surgery and to define safe limits of intraoperative mean arterial pressure in various groups of patients, including the elderly and those with hypertension. Now that non-invasive methods of cerebral angiography are available it would also be useful to determine whether the presence of significant cerebrovascular disease predisposes to cerebral injury.

Pamela J Shaw

DAVID BATES

NiALl E F CARTLIDGE

David Heaviside

JOYCE M FRENCH

DESMOND G JULIAN

DAVID A SHAW

Departments of Neurology and Cardiology,

University of Newcastle upon Tyne,

and the Cardiothoracic Unit,

Freeman Hospital

Newcastle upon Tyne

1 Sotaniemi KA, Mononen H, Hokkanen TE. Long-term cerebra outcome after open-heart surgery. A five year neuropsychological follow-up study. Stroke 1986;17:410-6.

Smith PLC, Treasure T, Newman SP, et al. Cercbral conscyuences of cardiopulmonary bypass. Lancet 1986 ; i:823-5.

Sotaniemi KA. Interhemispheric differences in tolerating extracorporeal circulation. Acta Neurol Scand 1982;65:166-7.

4 Breuer AC, Furlan AJ, Hanson MR, et al. Central nervous system complications of coronary artery bypass graft surgery: prospective analysis of 421 patients. Stroke 1983;14:682-7.

\section{Cryptosporidium and diarrhoea}

SIR,-For a consultant paediatrician to encourage bacteriologists to reduce the number of times they report "stool culture negative" by looking for cryptosporidium in patients with diarrhoea is indeed a clarion call. There must be few bacteriologists worth their salt who are not already complying, at least when the clinical information encourages them to do so. Dr J G Bissenden (2 August, $p$ 287) could have begun with abdominal pain and vomiting as major symptoms: both sometimes occur without diarrhoea, vomiting being a major presenting feature in over $40 \%$ of cases. ${ }^{1}$

Although some workers have reported an association with Giardia lamblia, ${ }^{1-3}$ others have not confirmed this association. ${ }^{4}$ The more common association with Campylobacter $\mathbf{s p}^{15}$ is not mentioned.

Dr Bissenden's comparison of staining methods is invalid: the true vardstick is total handling time, not staining time. ${ }^{6} \mathrm{He}$ fails to mention fluorescence staining by auramine, ${ }^{67}$ which is particularly reliable. ${ }^{8}$ It may be true to say that the number of oocysts present may sometimes be small: in acute cases they are usually seen in large numbers. When 